\title{
ON THE TIME-OPTIMALITY OF BANG-BANG TRAJECTORIES IN $\mathbf{R}^{3}$
}

\author{
HEINZ M. SCHAETTLER
}

1. Introduction. We study the problem of time-optimal control for a system

$$
\Sigma: \dot{x}=f(x)+u \cdot g(x), \quad|u| \leq 1, x \in \mathbf{R}^{3}, u \in \mathbf{R}
$$

where $f$ and $g$ are smooth vector fields. Admissible controls are arbitrary measurable functions with values in $[-1,+1]$ and a trajectory of the system corresponding to a control $u(\cdot)$ is an absolutely continuous curve such that $\dot{x}(t)=f(x(t))+u(t) g(x(t))$ holds almost everywhere.

In every optimal control problem the question of regularity of the solutions comes up naturally. The standard existence theorems only prove the existence of an optimal solution within the class of measurable functions. Necessary conditions for optimality put certain restrictions on optimal controls, in particular on their regularity properties. But, in general, they do not exclude a pathological behavior of optimal controls. In principle, the sets of discontinuities of an optimal control could even be a Cantor-like set with positive measure. On the other hand, certain regularity properties are necessary to construct a regular synthesis, i.e. to obtain sufficient conditions. For this one of the essential properties needed is that for every compact set $K$ there exists an integer $N=N(K)$ such that any time-optimal trajectory that lies in $K$ is a concatenation of at most $N$ "nice" pieces [3].

For our problem "nice" simply means trajectories corresponding to the constant controls $u=+1$ and $u=-1$ (bang arcs) or to a singular control (singular arc), which is a control usually with values in the interior of the control set and which satisfies certain compatibility conditions. (These are the possible candidates for time-optimal controls to which the necessary conditions of the Pontryagin maximum principle lead.)

For smooth single-input control-linear systems in the plane Sussmann showed that generically every point has a neighborhood $U$ such that timeoptimal trajectories that lie in $U$ are finite concatenations of bang and singular arcs with a bound on the number of pieces [4]. This was the key element in the proof of the existence of a regular synthesis for basically arbitrary analytic systems of this form in the plane $[5,6]$. A major issue in the proof of these results is to exclude the optimality of bang-bang trajectories with a large number of switchings in small times. (A bang-bang trajectory is a concatenation of bang arcs.) Since Sussmann's argument depended heavily on being in $\mathbf{R}^{2}$, even for the three-dimensional case so far only partial results

Received by the editors April 30, 1986 and, in revised form, October 9, 1986.

1980 Mathematics Subject Classification (1985 Revision). Primary 49B10; Secondary $93 \mathrm{~B} 10$.

Supported by an Alfred P. Sloan Doctoral Dissertation Fellowship 1985/1986. 
are known, due to Bressan [2] and Bonnard [1]. Here we announce a result which in a certain sense covers the least degenerate cases possible in $\mathbf{R}^{3}$. The proof will appear elsewhere [7].

2. The result. We identify $\Sigma$ with the $C^{\infty} \operatorname{map} \Sigma=(f, g): \mathbf{R}^{3} \rightarrow \mathbf{R}^{6}$ and we equip $C^{\infty}\left(\mathbf{R}^{3}, \mathbf{R}^{6}\right)$ with the Whitney $C^{\infty}$ topology. Let $A$ be the open subset of systems $\Sigma$ for which the vectors $f, g$ and $[f, g]$ are independent everywhere. Then we have

THEOREM. For a generic system $\Sigma \in \mathcal{A}$ every point has a neighborhood $U$ such that a bang-bang trajectory that lies in $U$ and has more than seven switchings is not time-optimal.

We will give a sketch of the proof (without any technical details) in the least degenerate case. Let $p \in \mathbf{R}^{3}$ and assume that $f(p), g(p)$, and $[f, g](p)$ are independent. We let $X=f-g$ and $Y=f+g$ and we express the third order brackets of $X$ and $Y$ as linear combinations of $X, Y$, and $[X, Y]$.

$$
\begin{gathered}
{[X,[X, Y]]=a_{1} X+a_{2} Y+a_{3}[X, Y]=\alpha f+\cdots,} \\
{[Y,[X, Y]]=b_{1} X+b_{2} Y+b_{3}[X, Y]=\beta f+\cdots .}
\end{gathered}
$$

In the least degenerate case we also assume that $\alpha(p) \neq 0$ and $\beta(p) \neq 0$. We claim that $p$ has a neighborhood $U$ such that bang-bang trajectories that lie in $U$ and have more than two switchings are not time-optimal.

Let $\Gamma$ be a $Y X Y X$-trajectory, i.e. a concatenation of a $Y$-arc, followed by a $X$-trajectory, another $Y$-arc, and one more $X$-arc. We call the initial point $\tilde{p}$ and the switching points $p_{0}, p_{1}$ and $p_{2}$. Let $\tau_{1}$ and $\tau_{2}$ be the times along the intermediate $X$ and $Y$ arcs respectively. We want to exclude the optimality of $\Gamma$ beyond the third switching point $p_{2}$. To do so, we compare $\Gamma$ to another bang-bang trajectory of the form $Y X Y$ which steers $\tilde{p}$ to some point on $\Gamma$ just after $p_{2}$. It is possible to set up such a variation along $\Gamma$ since the equation

$$
p_{0} e^{\tau_{1} X} e^{\tau_{2} Y} e^{\tau_{1} s X}=p_{0} e^{t_{1} Y} e^{t_{2} X} e^{t_{3} Y}
$$

is solvable by smooth functions $t_{1}, t_{2}, t_{3}$ of $\tau_{1}, \tau_{2}, s$ near the origin. (We use exponential notation for the flows of vector fields and we let the diffeomorphisms act on the right.) It turns out that the solutions $t_{i}$ are positive functions provided $\tau_{1}$ and $\tau_{2}$ are positive. Therefore we get a trajectory of our system. We are interested in the difference of the times. Let

$$
\Delta\left(s ; \tau_{1}, \tau_{2}\right)=t_{1}+t_{2}+t_{3}-\tau_{1}-\tau_{2}-\tau_{1} s .
$$

LEMMA. If $\Gamma$ is time-optimal, then necessarily $\dot{\Delta}\left(0 ; \tau_{1}, \tau_{2}\right)=0$ and $\dddot{\Delta}\left(0 ; \tau_{1}, \tau_{2}\right) \geq 0$, where the dot stands for differentiation with respect to $s$.

The first relation follows if we differentiate $(*)$ with respect to $s$ and use the fact that the switching points $p_{0}, p_{1}$, and $p_{2}$ form a conjugate triple. This is merely a consequence of the Pontryagin maximum principle. The inequality is then obvious.

To get applicable criteria, we compute an asymptotic expansion for $\Delta$. We rewrite both sides of $(*)$ in terms of canonical coordinates of the second kind and then compare the coefficients. The result is

$$
\Delta\left(s ; \tau_{1}, \tau_{2}\right)=\tau_{1} \tau_{2}\left\{-\frac{1}{2} s\left(\alpha\left(p_{0}\right) \tau_{1}+\beta\left(p_{0}\right) \tau_{2}+O(2)\right)+\frac{1}{2} s^{2}\left(\beta\left(p_{0}\right) \tau_{2}+O(2)\right)\right\}
$$


Here $O(2)$ stands for terms of order $\geq 2$ in the variables $\tau_{1}$ and $\tau_{2}$. So we get as necessary conditions near $p$

(I) $\alpha\left(p_{0}\right) \tau_{1}+\beta\left(p_{0}\right) \tau_{2}+O(2)=0$,

(II) $\beta\left(p_{0}\right) \tau_{2}+O(2) \geq 0$.

By assumption $\alpha(p)$ and $\beta(p)$ do not vanish and so these terms are large relative to the times $\tau_{i}$ on a sufficiently small neighborhood of $p$. (Since $f(p)$ and $g(p)$ are independent, we can assume that the times are small. The reason is that, once a time $T$ has been fixed, then we can shrink $U$ so that every trajectory of the system has to leave $U$ within time $T$. Then our analysis applies on $U$.) Therefore it is clear that (I) cannot hold near $p$ if $\alpha(p)$ and $\beta(p)$ have the same sign. If $\alpha(p)$ and $\beta(p)$ have opposite signs, then (I) need not be violated, but it implies that the times $\tau_{1}$ and $\tau_{2}$ are comparable (i.e. bounds $\tau_{1} \leq C \tau_{2}$ and $\tau_{2} \leq C \tau_{1}$ hold, where $C$ is a constant depending only on a neighborhood of $p$, but not on the individual trajectory). Therefore, if $\beta(p)<0$, then (II) excludes the optimality of $\Gamma$ as well.

If $\alpha(p)<0$ and $\beta(p)>0$, then these conditions do not help. However, it turns out that this case is actually the easier one. Here we can even exclude the optimality of the $Y X Y$-concatenation with switching points $p_{0}$ and $p_{1}$. To do this, we show that $\mathbf{R}^{3}$ is an approximating cone $\tilde{K}$ at $\tilde{q}$ for the set of points reachable from $\tilde{p}$ within time $\tilde{T}$, where $\tilde{q}=p_{1} e^{r Y}$ is a point on $\Gamma$ just after $p_{1}$ and $\tilde{T}$ is the time along $\Gamma$ from $\tilde{p}$ to $\tilde{q}$. This implies then that $\Gamma$ is not time-optimal beyond $p_{1}$.

The convex cone $\tilde{K}$ is generated by the vectors

$$
\begin{gathered}
-f(\tilde{q}), \quad-g(\tilde{q}), \quad e^{-r \cdot \operatorname{ad} Y}\left(g\left(p_{1}\right)\right), \\
e^{-r \cdot \operatorname{ad} Y}\left(-[X, Y]\left(p_{1}\right)\right) \quad \text { and } e^{-r \cdot \operatorname{ad} Y} e^{-\tau_{1} \text { ad } X}\left([X, Y]\left(p_{0}\right)\right) .
\end{gathered}
$$

The first three vectors are variational vectors which arise when we delete time or when we interchange $X$ and $Y$. The last two are variational vectors which can only be obtained at the bang-bang junctions via a variation of Lie type such as

$$
v(s)=p_{1} e^{-s X} e^{s Y} e^{s X} e^{-s Y}=p_{1} \exp \left(-\frac{1}{2} s^{2}[X, Y]+O\left(s^{3}\right)\right) .
$$

This leads to $-[X, Y]\left(p_{1}\right)$ and then we transport this vector to $\tilde{q}$. Similarly we obtain $[X, Y]\left(p_{0}\right)$ from the $Y X$-junction, which leads to the last vector. If now $r=0$, then we have

$$
e^{-\tau_{1} \operatorname{ad} X}\left([X, Y]\left(p_{0}\right)\right)=[X, Y]\left(p_{1}\right)+O\left(\tau_{1}\right)
$$

and the vector

$$
\begin{aligned}
e^{-\tau_{1} \text { ad } X}\left([X, Y]\left(p_{0}\right)\right)-[X, Y]\left(p_{1}\right) & =\tau_{1}\left([X,[X, Y]]\left(p_{1}\right)+O\left(\tau_{1}\right)\right) \\
& =\tau_{1}\left(\left(-\alpha\left(p_{1}\right)+O\left(\tau_{1}\right)\right) f\left(p_{1}\right)+\cdots\right)
\end{aligned}
$$

has a positive $f$-coefficient. Therefore, for $r=0$, these vectors generate $\mathbf{R}^{3}$ as convex cone and this does not change for small $r$.

This excludes the optimality of $Y X Y X$-trajectories. Using an input symmetry of $\Sigma$ which interchanges $X$ and $Y$, it follows that also $X Y X Y$-trajectories are not time-optimal near $p$. This proves the claim. 
For more degenerate generic cases (i.e. when $\alpha(p)$ and/or $\beta(p)$ vanish), we need the higher-order terms in the asymptotic expansion for $\Delta$ and we also need more terms in the relation $-\alpha\left(p_{1}\right)+O\left(\tau_{1}\right)$ which is crucial for the approximating cone argument. For bang-bang trajectories with more switchings we then combine conditions to obtain applicable criteria for these cases as well. This becomes highly technical and we do not even attempt to outline the arguments here.

\section{REFERENCES}

1. B. Bonnard, On singular extremals in the time minimal control problem in $R^{3}$, SIAM J. Control Optim. 23 (1985), 794-802.

2. A. Bressan, The generic local time-optimal stabilizing controls in dimension 3, SIAM J. Control Optim. 24 (1986), 177-190.

3. P. Brunovsky, Existence of regular synthesis for general control systems, J. Diff. Equations 38 (1980), 317-343.

4. H. Sussmann, The structure of time-optimal trajectories for single-input systems in the plane: The $C^{\infty}$ nonsingular case, SIAM J. Control Optim. (to appear).

5. - The structure of time-optimal trajectories for single-input systems in the plane: The general real analytic case, SIAM J. Control Optim. (to appear).

6. ___ Regular synthesis for time-optimal control of single-input real analytic systems in the plane, SIAM J. Control. Optim. (to appear).

7. H. Schaettler, On the local structure of time-optimal bang-bang trajectories in $R^{3}$ (to appear).

Department of Mathematics, Rutgers University, NeW Brunswick, NEW JERSEY 08903 